\title{
МЕТОДЫ ХИРУРГИЧЕСКОГО ЛЕЧЕНИЯ МНОГОУЗЛОВОГО ЗОБА У ПАЦИЕНТОВ В ЙОДОДЕФИЦИТНОМ РЕГИОНЕ
}

\section{METHODS OF SURGICAL TREATMENT OF MULTINODULAR GOITER IN PATIENTS LIVING IN AN IODINE-DEFICIENT REGION \\ M. Magomedov \\ O. Osmanov}

Summary. The results of surgical treatment of 548 patients with multinodular goiter were analyzed over a ten-year period. The middle age of the examined patients was $50.3+11.6$ years, $80 \%$ of women. The study objective is to determine the risk factors for complications and their occurrence post various methods of thyroidectomy in patients with multinodular goiter. The patients were divided into two groups. The patients operated in 2009-2015 (308 patients in total) were assigned to the first group with subtotal thyroidectomy. The second group included 240 patients who underwent total thyroidectomy in 2016-2019. Among 308 patients of the first group, 85 patients (27.5\%) had relapse and 139 patients (41.9\%) had hypothyroidism. When comparing the surgical procedures such as subtotal thyroidectomy and total thyroidectomy, statistical significance was registered. Transient hypoparathyroidism was diagnosed much more often in patients post total thyroidectomy vs patients post subtotal thyroidectomy.

The key factors affecting the recurrence of benign thyroid nodule are: young age, iodine deficiency in the region, bilateral multinodular goiter and insufficient surgical intervention. If we consider the methods of surgical treatment of multinodular goiter with regard to the risk of complications, then total thyroidectomy is most effective in comparison with subtotal thyroidectomy.

Keywords: multinodular goiter, thyroidectomy, subtotal thyroidectomy, hemithyroidectomy, thyroid gland.

\section{Вве $А$ ение}

B опросы выбора метода хирургического лечения многоузлового зоба в настоящее время представляют собой актуальную проблему, в связи с увеличением количества таких пациентов и отсутствием единых подходов к определению оперативной тактики [1-2]. Тиреоидэктомия остается важным вариантом лечения многоузлового зоба и является одной из самых часто выполняемых операций хирургами общей прак-
Магомедов Мухума Магомедович

Д.м.н., профеессор, ФГБОУ ВО «Дагестанский государственный медицинский университет» МЗ РФ

(Махачкала)

muxua@mail.ru

Османов Омар Магомедтагирович

К.м.н., ГБУ «Городская клиническая больница № 67»

(Москва)

omarov@mail.ru

Аннотация. Анализированы результаты оперативного лечения 548 пациентов с многоузловым зобом за 10 лет. Средний возраст обследованных составил 50,3+11,6 лет, 80\% женщин. Целью настоящего исследования является определение факторов риска осложнений и их возникновения после применения различных методов тиреоидэктомии у пациентов с многоузловым зобом. Все пациенты были разделены на две группы. Больные оперированные за 2009-2015 годы в количестве 308 пациентов были отнесены к первой группе с субтотальным удалением щитовидной железы. Во второй группе были отнесены 240 пациентов оперированные за период 2016-2019 годы тотальным удалением щитовидной железы. Из 308 пациентов первой группы был рецидив у 85 (27,5\%) и гипотиреоза у 139 (41,9\%) случаях. При сравнении таких хирургических процедур, как субтотальная тиреоидэктомия и тотальная тиреоидэктомия была зафиксирована статистическая значимость. Транзиторный гипопаратиреоз диагностировался гораздо чаще у пациентов после проведения тотальной тиреоидэктомии по сравнению с пациентами, перенесшими субтотальную тиреоидэктомию.

Основными факторами, влияющими на рецидив доброкачественного узлового образования щитовидной железы, являются: молодой возраст, йододефицитность региона, двусторонний многоузловой 306 и недостаточное хирургическое вмешательство. Если рассматривать методики хирургического лечения многоузлового зоба с позиции степени риска возникновения осложнений, то использование тотальной тиреоидэктомии наиболее эффективно по сравнению с субтотальной тиреоидэктомией.

Ключевые слова: многоузловой зоб, тиреоидэктомия, субтотальная тиреоидэктомия, гемитериоидэктомия, щитовидная железа.

тики за рубежом. Дагестан является эндемичным регионом, где у большинства пациентов диагносцируется многоузловой зоб [3]. Несмотря успехи современной эндокринной хирургии, количество послеоперационных осложнений остается высоким - 6-11\%, что обусловлено различными подходами к определению показаний к операции, недостаточный технический вариант общих хирургов и их подготовка [4]. Как известно, оперативное лечение многоузлового зоба, часто осложняется с повреждением анатомических структур шеи, наибольшее 
значение имеют возвратные гортанные нервы, транзиторную гипокальцемию и постоянный гипопаратириоз [5].

В последнее время операция по удалению доброкачественного зоба была признана приемлемым и безопасным методом лечения, с низким хирургическим риском и смертностью [6-3]. Несмотря на улучшение диагностики и лечения заболеваний щитовидной железы, в последние два десятилетия в литературе остается спорным вопрос о том, какой метод эффективней [7]. Ранее субтотальная тиреоидэктомия при многоузловом зобе рассматривалась как лечение по золотому стандарту, что было обусловлено сокращением случаев осложнений со стороны гортанного нерва и паращитовидной железы, уменьшением потребности в пожизненной заместительной гормональной терапии [8,7]. Известно, что при длительном наблюдении после субтотальной тиреоидэктомии при многоузловом зобе следует ожидать увеличения частоты рецидивов до 78\% [9,2]. Вместе с тем не секрет, что повторная операция при рецидиве зоба приводит к увеличению риска повреждения верхнего гортанного нерва и паращитовидной железы. В настоящее время многие клиники изменили свою оперативную тактику в пользу применения тотальной тиреоидэктомии, хотя и субтотальная тиреоидэктомия по-прежнему остается предпочтительным вариантом [10].

Оценка эффективности радикальных и органосохраняющих оперативных вмешательство при многоузловом зобе с точки зрения отдаленных результатов хирургического лечения, неоднозначна, поэтому, актуальным остается поиск оптимальных методик лечения многоузлового зоба. Руководствуясь выше приведенными данными, была поставлена цель оценить результативность применения различных методов операций при диагностированном многоузловом зобе.

\section{Цель исслеАования}

Определить факторов риска осложнений и их возникновения после операции на щитовидной железе у пациентов многоузловым зобом.

\section{Материалы и метолы}

Исследование было одобрено Этическим комитетом Дагестанского государственного медицинского университета. Пациенты дали согласие на обработку персональных данных. Проведен ретроспективный анализ в общей сложности у 548 пациентов, которым с 2009 по 2019 годы были выполнены операции на щитовидной железе с диагнозом многоузлового зоба доброкачественной этиологии. Все пациенты были разделены на две группы. Пациенты оперированные за 2009-2015 годы в количе- стве 308(56,2\%) были отнесены к первой группе. Пациентам проводилась субтотальная тиреоидэктомия (СТ), необходимость манипуляции была основана на результатах предоперационных тестов, интраоперационного исследования и анализа гистологических результатов. Во второй группе были отнесены 240 (43,8\%) пациентов оперированные за период 2016-2019 годы с проведением тотальным удалением щитовидной железы.

Были собраны и изучены данные у ранее прооперированных (48\%) пациентов - возраст, пол, семейный анамнез, применение заместительной терапии L-тироксином, симптоматика, пред- и послеоперационным лечение, частота рецидивов, осложнений, опыт хирурга. Информация была собрана из истории болезни, операционных данных, результатов гистологического исследования и физикального обследования. Также был оценен уровень тиреоидных гормонов в сыворотке крови, состояние тиреоидного остатка. Пациентам было проведено ультразвуковое исследование щитовидной железы на аппарате Aloka SSD5000 (фирма Япония) с использованием конвексных датчиков с частотой 5 МГц. На УзИ определяли наличие и объем оставшейся ткани щитовидной железы, а также признаки узловых образований щитовидной железы. Также было назначено дополнительное обследование у отоларингологов. Показанием к выявлению рецидива было наличие узлов с риском развития злокачественности, большого зоба и компрессионных симптомов. Для каждой операции рассчитывали частоту рецидивов и количество пациентов, нуждающихся в повторной операции. Пациенты первой группы были разделены на две подгруппы: 1-ю подгруппу составили пациенты без рецидива заболевания, во 2-ю подгруппу вошли пациенты, у которых рецидив был выявлен в процессе наблюдения.

Подробно изучались имеющиеся в анамнезе послеоперационные осложнения, включавшие транзиторную гипокальциемию, перманентный гипопаратиреоз, рецидивирующее повреждение гортанного нерва и послеоперационная гематома. Гипокальциемия определялась по уровню кальция в сыворотке крови с содержанием менее 8,4 мг/дл (нормальный диапазон: 8,4-10,4 мг/дл). Транзиторная гипокальциемия определялась как послеоперационная гипокальциемия, которая разрешилась в течение 6 месяцев после тиреоидэктомии. Перманентный гипопаратиреоз определялся, как послеоперационная гипокальциемия, сохраняющаяся в течение более чем 6 месяцев после операции. В отдельных случаях проводил КТ исследования для верификации загрудинного рецидивного зоба.

Статистический анализ проводился за счет SPSS для Windows (SPSS Inc. Chicago IL, USA), версия 15.0. Результаты определились в виде среднего значения 
\pm стандартное отклонение (SD). Связь между клиническими данными и рецидивом заболевания или необходимостью повторного вмешательства анализировали с помощью точного критерия Фишера (для категориальных переменных) и U-критерия Манна-Уитни (для непрерывных переменных). Достоверным считали $\mathrm{p}<0,05$.

\section{Результаты}

Пациенты дали информированное согласие на представление, хранение, анализ полученных данных. Средний возраст 548 обследованных пациентов составил $50,3 \pm 11,6$ года, $80 \%$ из которых женщины. За период 2010-2015 г. 308 (56,2\%) пациентам была назначена СТЭ (первая группа), начиная с 2010 и по 2015 г. Во второй группе были отнесены 240 (43,8\%), которые перенесли ТТЭ. Тиреоидэктомия была хирургической процедурой выбора для всех пациентов второй группы. Наиболее важные принципы, используемые при выполнении операции на щитовидной железе было: 1) визуализация возвратных гортанных нервов и околощитовидных желез; 2) попытка определить наружные ветви верхнее гортанного нерва перед перевязкой верхней щитовидной артерии; 3) перевязки ветвей нижней щитовидной артерии для сохранения кровоснабжения паращитовидных желез. Изменение в оперативном лечении было сделано в ответ на выявлении частых рецидивов многоузловом зобе. Частота рецидива зоба первой группы составила $27,5 \%$ (n =85) и гипотиреоза у $139(45,1)$ пациентов. Интервал между первичной операцией и выявленным рецидивом составил 4,8 22 года (диапазон 2-8 лет) и спустя 10 лет с момента операции рецидив развился и еще у 12 пациентов. Частота рецидива второй группы не было.

Статистически значимых различий в сравнении возраста, пола и семейного анамнеза между группами не выявлено ( $p>0,05)$.

В 1-й группе было больше пациентов, получавших заместительную терапию L-тироксином, который не оказал влияния на частоту рецидивов.

В группе оперированных не было зафиксировано летальных случаев. Два пациента $(0,8 \%)$ второй группы нуждались в срочном повторном оперативном вмешательстве по поводу гематомы. Среднее время операции составило первой группе $(120+12,1$ мин, (диапазон 58185 мин). Во второй группе составило 139+25,3 мин, (диапазон 70-212 мин). Речевые нарушения была зафиксирована у $1(0,4 \%)$ пациента, у которых послеоперационной фиброоптической видиоларингоскопии был визуализирован односторонный паралич наружного гортанного нерва. Не было зафиксировано ни одного случая дву- сторонней травмы нерва. Парезы гортанны были отмечены у 10 (4,7\%) и транзиторная гипокальцимия у 17 $(7,1 \%)$ пациентов, а гипотиреоз у всех оперированных. Из 308 (56,2\%) оперированных пациентов первой группы осложнения возникли наиболее часто, рецидив у 85 (27,6\%), парез гортани у 12 (3,8\%) пациентов, у 13 (4,2\%) транзиторная гипокальцемия и гипотиреоз у 129(41,8\%). Перманентного гипопаратиреоза и транзиторного или перманентно рецидивирующего паралича гортанного нерва достоверно не различались между больными после проведенных СТЭ и ТЭ ( $>$ > 0,05), в то время как транзиторный гипопаратиреоз был достоверно выше у больных ТЭ по сравнению с пациентами СТЭ $(\mathrm{p}<0,05)$. Повторная госпитализация потребовалась $(0,7 \%)$ пациентам спустя 5 суток из-за гематомы у пациентов второй группы.

\section{Обсужление}

Границы резекции при операциях на щитовидной железе менялись годами. До конца XX века тотальная тиреоидэктомия, за исключением рака щитовидной железы, выполнялась крайне редко из-за высокой частоты осложнений. В этот период именно субтотальная тиреоидэктомия при доброкачественных заболеваниях щитовидной железы была принята в качестве золотого стандарта терапии. Однако после длительного наблюдения за результатами после проведения субтотальной тиреоидэктомии было очевидно, что эта операция приводит к частым рецидивам. После этого начались дискуссии о наиболее оптимальном методе хирургического вмешательства при доброкачественных заболеваниях щитовидной железы [11, 3].

Большинство исследований, связанных с изучением рецидива при многоузловом зобе, проводятся после 4-5 лет послеоперационного периода. В случае более длительного периода наблюдения закономерно возникновение увеличения частоты рецидивов. Частота рецидивов после СТЭ, особенно после наблюдения в течение 10 лет, достигает 40\% [12]. Рецидивы доброкачественных заболеваний щитовидной железы определяются в литературе по-разному [13,5]. Инструментами, для выявления рецидива являются: физикальный осмотр, УЗИ щитовидной железы, функциональные тесты щитовидной железы, сцинтиграфия, а иногда и компьютерная томография.

Основной целью хирургического лечения заболеваний щитовидной железы всегда должно быть выполнение наиболее эффективного лечения с минимальными осложнениями и частотой рецидивов. Тиреоидэктомия при доброкачественных заболеваниях устраняет заболевание и предотвращает возможность рецидива. Однако авторы, которые выступают против тотальной 
тиреоидэктомии при лечении доброкачественных заболеваний, отмечают повышенный риск возникновения рецидива, в том числе поражение гортанного нерва и паращитовидной железы [14,6]. Они подчеркивают, что эти пациенты также приговорены к пожизненной заместительной гормональной терапии. В настоящем исследовании после первичного хирургического вмешательства показатели осложнения паралича гортанного нерва и паращитовидной железы были не выше, чем сообщалось в литературе [15,9]. Кроме того, только транзиторная гипокальциемия была достоверно выше у больных, которым проводилась ТЭ по сравнению с больными после СТЭ.

Хирургическое лечение во время повторных операций затруднено из-за фиброза, возникшего в результате проведения операции.

Заболеваемость, вызванная травмой нерва, в этой ситуации выше. Развитие паралича ВТН при повторной операции напрямую связано с опытом хирурга и составляет от 0 до 14\%, что отмечено в литературе [12,2]. Рецидивирующий паралич гортанного нерва после предыдущей операции может маскироваться контралатеральной компенсацией, и голос может казаться нормальным. Таким образом, предоперационное обследование голосовых связок должно проводиться у пациентов, которые являются кандидатами на повторную операцию, и в случае предполагаемого риска, некоторая часть ткани щитовидной железы должна быть оставлена в задней части, чтобы предотвратить двустороннее повреждение нерва.

Перед повторной операцией оценить локализацию и функциональное состояние паращитовидных желез порой невозможно. Выделение паращитовидных желез может быть затруднено, и для предотвращения постоянной гипокальциемии при случайно удаленных железах рекомендуется аутотрансплантация $[8,9]$. Даже когда повторные операции выполняются опытными хирургами, риск повреждения паращитовидной железы и ВГН значительно выше по сравнению с первой операцией. Мы провели сравнительное исследование между повторной операцией щитовидной железы и первичной операцией щитовидной железы с точки зрения осложнений. В повторных случаях наблюдается значительно более высокая степень осложнений с повреждением ВГН и паращитовидной железы.

В последнее время исследования, касающиеся рецидива при доброкачественных заболеваниях щитовидной железы, показывают, что молодой возраст, мультинодулярный зоб, йододефицитный регион, семейный анамнез и неадекватная хирургия являются основными факторами риска рецидива. Для предотвращения рецидива доброкачественного заболевания щитовидной железы необходима детальная предоперационная оценка, чтобы решить вопрос о соответствующем хирургическом вмешательстве. Качество ультразвукового исследования с тонкоигольной биопсией, которое используется как для доброкачественных, так и для злокачественных заболеваний щитовидной железы, зависит от квалификации врача ультразвуковой диагностики и хирурга. При многоузловом зобе 40\% узлов расположены близко к задней капсуле щитовидной железы и могут быть оставлены во время субтотальной резекции [7,12]. Эти узлы могут расти независимо от ТТГ и приводить к высокой частоте рецидивов. В частности, в эндемичных регионах такие процедуры, как энуклеация, субтотальные и гемитиреоидэктомия, не рекомендуются при многоузловом зобе, поскольку существует неизбежный риск распространения рецидива на оставшейся ткани $[8,13]$. Тотальная тиреоидэктомия рассматривается как все более предпочтительный вариант лечения узловых заболеваний, вовлекающих обе доли щитовидной железы при доброкачественных зобах.

Семейный анамнез может рассматриваться как существенный фактор в развитии рецидива после операции по поводу многоузлового зоба $[14,15]$. В проведенном исследовании не было выявлено достоверных различий при сравнении семейного анамнеза между группами $(p>0,05)$.

Некоторые исследования показали, что терапия L-тироксином защищает от рецидивов и повторных операций, уменьшая стимуляцию ТТГ на остаточной ткани. Это позволяет утверждать, что добавление тиреоидных гормонов после консервативной операции может эффективно защитить пациентов от рецидива $[10,11]$. Но, с другой стороны, по данным других исследователей, несмотря на заместительную терапию, длительное послеоперационное наблюдение показало высокую частоту рецидивов после субтотальной тиреоидэктомии [4,7]. Недавние исследования показывают, что защитный эффект гормональной терапии является спорным $[2,7,10]$.

\section{Зак^ючение}

Ограниченные хирургические резекции, отсутствие заместительной терапии, йододефицитный регион, молодой возраст должны учитываться в качестве сопутствующих факторов рецидива. Необходимость повторной операции при рецидивах доброкачественных заболеваний щитовидной железы является серьезной клинической проблемой в связи с высокой частотой послеоперационных осложнений. Фактически можно утверждать, что тотальная тиреоидэктомия является предпочтительной операцией при многоузловом зобе, во избежание возникновения рецидива. 


\section{ЛИТЕРАТУРА}

1. Кахаров А. М., Ибодов Г. К. Современные аспекты диагностики и хирургического лечения доброкачественного узлового и многоузлового зоба ІІЗдравоохранения Таджикистана. 2016. № 1. С. 77-82.

2. Кухненко Ю. В., Косивцов 0. А., Михин И. В., Рясников Л. А. Результаты хирургического лечения пациентов с различными заболеваниями щитовидной железы // Весник ВолГМУ. 2015. Том 4. № 56. С. 67-72.

3. Магомедов М. М., Адыкбекова 3. А. Заболевание щитовидной железы ее осложнения. Махачкала «Лотос», 2020. С. 146.

4. Aleksander P. E., Bruckner-Spieler M., Stoehr A. M., Lankes E., Kiihnen P., Schnabel D., Emert A., Stablein W, Craig M. E., Blankenstein 0., et al. Mean high-close 1-thyroxine treatment is efficient and safe to achieve a normal IQ in young adult patients with congenital hypothyroidism. Journal of Clinical Endocrinology and Metabolism. 2018. vol.103. p.1459-1469. (10.1210/jc.2017-01937).

5. Dreval A.V. Hypothyroidism. In: Karachentsevlul, Kazakov A.Y, Kravchun N. A., IPina M., editors. 100 izbrannykhlektsii po endokrinologii [100 selected lectures on endocrinology], 2nd ed. Kharkiv: SAM. 2014. p.1000.

6. Jonklaas J., Bianco A. C., Bauer A. J., Burman K. D., Cappola A. R., Celi F. S., Cooper D. S., Kim B. W„, Peeters R. P., Rosthal M. S., et al. Guidelines for the treatment of hypothyroidism: prepared by the American Thyroid Association task force on thyroid hormone replacement. Thyroid. 2014. vol. 24. p.1670-1751. (10Л089/ thy.2014.0028).

7. Mohammed N. S., Ahmed S. A. Postoperative complications after total thyroidectomy for denign thyroid diseases. ISJ.2019:6(9):3064-3068. D0I: 10.18203/23492902.isj20194034.

8. Mok V. M., Oltmann S. C., Chen H., Sippel R. S., Schneider D. F. Identifying predictors of a difficult thyroidectomy. J. Surg. Res. 2014. vol. 190. p. $157-163$.

9. Pankiv V. I. Thyroid-stimulating hormone level in blood as a basic diagnostic marker and criterion of successful treatment of thyroid diseases. Reproductive Endocrinology. 2017. vol. 3. no. 35. p. 84-88. (in Ukrainian).

10. Yasin Peker, Necat Cin, Haldun Kar, Fatma Tar [et.al.] Prospective Evaluation of Perioperative Biochemical Test to Predict Hypocalcemia After Total Thyroidectomy.2020. vol.82. p.187-190.

11. Persani L., Candiano B., Bonomi M. The diagnosis and management of central hypo thyroidism. I., 2018 // Endocrine connections, 2019. Vol. 8. p. $44-54$.

12. Qari F. Hypothyroidism in clinical practice. J Family Med Prim Care. 2014. vol. 3. no.2. p. 98-101. doi: 10.4103/2249-4863.137

13. Rayes N., Steinmiiller T., Schroder S., Klotzler A., Bertram H., Denecke T., et al. Bilateral subtotal thyroidectomy versus hemithyroidectomy plus subtotal resection (Dunhill Procedure) for benign goiter: long-term results of a prospective, randomized study. World J Surg. 2013. vol. 37 no.1. p. 84-90.

14. Shyam S. T., Ayathu VS., Sai. M. Solitary thyroid: an overview of one year study at a tertiary carehospital. ISJ. 2019. vol. 6(a). p. 3117-3121. D0I: http://dx.dof. org/10.18203/2349-2902. isj20193648.

15. Zambudio A. R., Rodriguez J., Riquelme J., Soria T., Canteras M., Parrilla P. Prospective study of postoperative complications after total thyroidectomy for multinodular goiters by surgeons with experience in endocrine surgery. Ann Surg. 2004. vol. 240. no.1. p. 18-25. 